\title{
Symbols and Abbreviations
}

For abbreviations of journals, monograph series, and individual works, see Bibliography.

I. People and places

$\begin{array}{llll}\text { AW } & \text { Anthony R. Walker } & \text { JHT } & \text { James Haxton Telford } \\ \text { CB } & \text { Cà-bí } & \text { JKW } & \text { Julian K. Wheatley } \\ \text { CC } & \text { Christopher Court } & \text { LG } & \text { Laurie Goldman } \\ \text { CM } & \text { Cà-mó } & \text { MCF } & \text { George B. McFarland } \\ \text { Cu } & \text { Josiah Nelson Cushing } & \text { PL } & \text { Paul Lewis } \\ \text { DB } & \text { David Bradley } & \text { RJL } & \text { Randy J. LaPolla } \\ \text { GY } & \text { Gordon Young } & \text { SEA } & \text { Southeast Asia } \\ \text { HT } & \text { Hwè Tà? village } & \text { VY } & \text { Vincent Young } \\ \text { HY } & \text { Harold Young } & \text { X'n } & \text { Christian } \\ \text { ILH } & \text { Inga-Lill Hansson } & \text { YP } & \text { Yâ-pāać }\end{array}$

JAM James A. Matisoff 
II. Language and dialect names

\begin{tabular}{|c|c|c|c|}
\hline Ak. & Akha & N. Thai & $\begin{array}{l}\text { Northern Thai } \\
\text { (= Kham Myang) }\end{array}$ \\
\hline BL & Black Lahu & & \\
\hline Bs. & Burmese & $O C$ & $\begin{array}{l}\text { Old Chinese } \\
(=\text { Archaic Chinese })\end{array}$ \\
\hline Ch. & Chinese & PLB & Proto-Lolo-Burmese \\
\hline Chin. & Chinese & PST & Proto-Sino-Tibetan \\
\hline Chin.Lh. & Chinese Lahu & $\mathrm{PT}$ & Proto-Tai \\
\hline Eng. & English & PTB & Proto-Tibeto-Burman \\
\hline Fr. & French & $\mathbf{R L}$ & Red Lahu \\
\hline Inscr. Bs. & Inscriptional Burmese & Si. & Siamese (= Standard Thai) \\
\hline Jg. & Jingpho & Skt. & Sanskrit \\
\hline Jse. & Japanese & ST & Sino-Tibetan \\
\hline LB & Lolo-Burmese & TB & Tibeto-Burman \\
\hline Lh. & Lahu & $\mathrm{TN}$ & Tai Nuea [Gedney 1976] \\
\hline Mand. & Mandarin & WB & Written Burmese \\
\hline MC & $\begin{array}{l}\text { Middle Chinese } \\
\text { (= Ancient Chinese) }\end{array}$ & $\mathbf{W T}$ & Written Tibetan \\
\hline Mod. Bs. & Modern Burmese & $\begin{array}{l}\text { Yid. } \\
\text { YL }\end{array}$ & $\begin{array}{l}\text { Yiddish } \\
\text { Yellow Lahu }\end{array}$ \\
\hline
\end{tabular}


III. Form Classes and Construction Types

\begin{tabular}{|c|c|c|c|}
\hline Adjintens & intensified adjective & $C l_{f}$ & final clause \\
\hline Adv & adverb & $C \ln f$ & non-final clause \\
\hline $\mathbf{A E}$ & adverbial expression & $\mathrm{Cln}+\mathrm{Cl}_{f}$ & $\begin{array}{l}\text { non-final clause plus } \\
\text { final clause }\end{array}$ \\
\hline 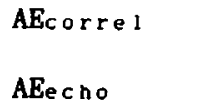 & $\begin{array}{l}\text { correlative adverbial } \\
\text { echo-adverbial }\end{array}$ & $C l_{\text {nom }}$ & nominalized clause \\
\hline$A E_{e} \times t$ & extentive adverbial & $\mathrm{Clnom}_{\mathrm{n}} \mathrm{ext}$ & $\begin{array}{l}\text { extentive nominalized } \\
\text { clause }\end{array}$ \\
\hline AEonomat & $\begin{array}{l}\text { onomatopoetic } \\
\text { adverbial }\end{array}$ & $\mathrm{Cln}_{\mathrm{n} \mathrm{m} / \mathrm{q}}$ & $\begin{array}{l}\text { quantified nomi- } \\
\text { nalized clause }\end{array}$ \\
\hline $\mathrm{AE}_{\mathbf{q}}$ & quantified adverbial & Clobj & clausal object \\
\hline AEqha & qha-adverbial & Clpurp & purpose clause \\
\hline$A E_{s} t a t$ & stative adverbial & Clquot & quotative clause \\
\hline AEs tat $+N_{h}$ & $\begin{array}{l}\text { noun-head modified by } \\
\text { a preposed stative } \\
\text { adverbial }\end{array}$ & $C l_{\text {spec }}+V_{h}$ & $\begin{array}{l}\text { specifying clause } \\
\text { plus main verb }\end{array}$ \\
\hline B & bound morpheme & $\mathrm{Cl}_{\mathrm{s}}$ ubj & clausal subject \\
\hline Badv & bound adverbial & $\mathrm{Cl}_{\mathrm{tag}}$ & clause-tag \\
\hline Be 1 ab & $\begin{array}{l}\text { bound constituent of } \\
\text { an elaborate } \\
\text { expression }\end{array}$ & Clf & $\begin{array}{l}\text { time clause; temporal } \\
\text { clause } \\
\text { classifier }\end{array}$ \\
\hline $\mathrm{B}_{\mathbf{n}}$ & $\begin{array}{l}\text { bound nominal } \\
\text { morpheme }\end{array}$ & $\begin{array}{l}\text { Conj } \\
\text { Det }\end{array}$ & conjunction \\
\hline $\mathbf{B}_{\mathbf{v}}$ & bound verbal morpheme & DO & direct object \\
\hline $\mathrm{C}_{\boldsymbol{r}}$ & resultative & & \\
\hline $\mathrm{Cl}$ & $\begin{array}{l}\text { complement } \\
\text { clause }\end{array}$ & Elab & $\begin{array}{l}\text { elaborate expression } \\
\text { ( } 4 \text { syllables; lst and } \\
3 r d \text {, or } 2 \text { nd and } 4 \text { th } \\
\text { are the same) }\end{array}$ \\
\hline $\begin{array}{l}\text { Cladv } \\
\text { Cladv }+V_{h}\end{array}$ & $\begin{array}{l}\text { adverbial clause } \\
\text { adverbial clause } \\
\text { modifying a main verb }\end{array}$ & Blabadv & $\begin{array}{l}\text { adverbial elaborate } \\
\text { expression }\end{array}$ \\
\hline Clext & extentive clause & Elabcouplet & $\begin{array}{l}\text { elaborate couplet } \\
\text { (the non-repeated } \\
\text { syllables in an Elab) }\end{array}$ \\
\hline$C l_{e x t}+C l_{f}$ & $\begin{array}{l}\text { extentive clause } \\
\text { modifying a final } \\
\text { clause }\end{array}$ & Elabdeverb & $\begin{array}{l}\text { deverbative elaborate } \\
\text { expression }\end{array}$ \\
\hline
\end{tabular}




\begin{tabular}{|c|c|c|c|}
\hline Elabe $x t$ & $\begin{array}{l}\text { extentive elaborate } \\
\text { expression }\end{array}$ & Ndeverb & $\begin{array}{l}\text { deverbal noun (de- } \\
\text { rived from a clause } \\
\text { containing only a VP) }\end{array}$ \\
\hline Elabn & $\begin{array}{l}\text { nominal elaborate } \\
\text { expression }\end{array}$ & Ne $x t$ & extentive noun \\
\hline ElabN-spec & $\begin{array}{l}\text { elaborate specifying } \\
\text { noun }\end{array}$ & $\mathrm{Nh}_{\mathrm{h}}$ & noun-head; head noun \\
\hline Elabq & $\begin{array}{l}\text { quantified elaborate } \\
\text { expression }\end{array}$ & $\mathrm{Nh}_{\mathrm{h}}+\mathrm{AE}_{\mathrm{stat}}$ & $\begin{array}{l}\text { noun-head modified by } \\
\text { a postposed stative } \\
\text { adverbial }\end{array}$ \\
\hline Elabt ime & $\begin{array}{l}\text { temporal elaborate } \\
\text { expression }\end{array}$ & Nint $z$ & interrogative noun \\
\hline Elabv & verbal elaborate & $\mathrm{N}_{1}$ im & limited noun \\
\hline Ext & extentive & $\mathbf{N}_{q} \mathbf{n}$ & $\begin{array}{l}\text { quantified head; } \\
\text { noun-head of a quan- }\end{array}$ \\
\hline $\begin{array}{l}\text { Extended } \\
\text { Elab }\end{array}$ & $\begin{array}{l}\text { extended elaborate } \\
\text { expression (more than }\end{array}$ & & tified NP \\
\hline Inter $j$ & $\begin{array}{l}4 \text { syllables) } \\
\text { interjection }\end{array}$ & $N_{r h}$ & $\begin{array}{l}\text { relative head; noun- } \\
\text { head of a relative } \\
\text { clause }\end{array}$ \\
\hline I0 & indirect object & $N_{r h / q}$ & $\begin{array}{l}\text { quantified relative } \\
\text { head }\end{array}$ \\
\hline LRC & $\begin{array}{l}\text { left-relative clause } \\
\text { (comes before head-- } \\
\text { noun) }\end{array}$ & $\mathrm{N}_{r h}+\mathrm{RRC}$ & $\begin{array}{l}\text { relative head modi- } \\
\text { fied by a right- } \\
\text { relative clause }\end{array}$ \\
\hline $\mathrm{LRC}+\mathrm{N}_{\mathrm{rh}}$ & $\begin{array}{l}\text { left-relative clause } \\
\text { modifying a relative } \\
\text { head }\end{array}$ & $\mathbf{N}_{\mathrm{s}} \mathrm{d}$ & $\begin{array}{l}\text { spatial-demonstrative } \\
\text { noun }\end{array}$ \\
\hline $\operatorname{Mpfx}$ & $\begin{array}{l}\text { prefixable morpheme } \\
\text { (may be preceded by }\end{array}$ & $\mathbf{N}_{\text {spec }}$ & specifying noun \\
\hline & j-) & Nspec/loc & $\begin{array}{l}\text { specifying locative } \\
\text { noun }\end{array}$ \\
\hline Mpfx/loc & $\begin{array}{l}\text { locative prefixable } \\
\text { morpheme }\end{array}$ & $\mathrm{N}_{\text {spec }}+\mathrm{V}$ & $\begin{array}{l}\text { noun specifying the } \\
\text { following verb }\end{array}$ \\
\hline$N$ & noun & & \\
\hline $\mathrm{Nadv}$ & adverbial noun; & & subject noun \\
\hline & "nadverb" & Nt ime & $\begin{array}{l}\text { noun of time; } \\
\text { temporal noun }\end{array}$ \\
\hline Ndeclaus & $\begin{array}{l}\text { declausal noun (de- } \\
\text { rived from a clause } \\
\text { containing a NP as }\end{array}$ & $\mathrm{N}_{\mathrm{t}}$ op ic & topic noun \\
\hline & well as a VP) & NP & noun phrase \\
\hline Ndesc & descriptive noun & $\mathrm{NP}_{\mathrm{conj}}$ & conjoined noun phrase \\
\hline & & NPdat & dative noun phrase \\
\hline
\end{tabular}




\begin{tabular}{|c|c|c|c|}
\hline NPdet & $\begin{array}{l}\text { determined noun } \\
\text { phrase }\end{array}$ & Pdev & deverbative particle \\
\hline NPext & extentive noun phrase & $P_{n}$ & noun particle \\
\hline NPext/gen & $\begin{array}{l}\text { genitival extentive } \\
\text { noun phrase }\end{array}$ & Pnom & $\begin{array}{l}\text { nominalizing particle } \\
\text { quotative particle }\end{array}$ \\
\hline $\mathrm{NP}_{\mathbf{f}}$ & final noun phrase & $\mathrm{Pu}_{u}$ & unrestricted particle \\
\hline NPgen & genitive noun phrase & Puf & $\begin{array}{l}\text { final unrestricted } \\
\text { particle }\end{array}$ \\
\hline NP1 oc & locative noun phrase & & \\
\hline NPn $f$ & non-final noun phrase & 1011 & stricted particle \\
\hline NPobj & object noun phrase & Puniv & $\begin{array}{l}\text { universal unre- } \\
\text { stricted particle }\end{array}$ \\
\hline NPpurp & $\begin{array}{l}\text { pronominal noun } \\
\text { phrase } \\
\text { purposive noun phrase }\end{array}$ & Puniv/nom & $\begin{array}{l}\text { nominalizing univer- } \\
\text { sal unrestricted } \\
\text { particle }\end{array}$ \\
\hline $\mathrm{NP}_{\mathbf{q}}$ & $\begin{array}{l}\text { quantified noun } \\
\text { phrase }\end{array}$ & $\mathbf{P}_{\mathbf{v}}$ & verb particle \\
\hline $\mathrm{NPq} /$ det & $\begin{array}{l}\text { determined quantified } \\
\text { noun phrase }\end{array}$ & $\begin{array}{l}\text { Pv/nom or } \\
\text { Pv-nom }\end{array}$ & $\begin{array}{l}\text { nominalizing } \\
\text { verb particle }\end{array}$ \\
\hline $\mathrm{NP}_{\mathbf{q} / \mathrm{t} \text { i me }}$ & $\begin{array}{l}\text { temporal quantified } \\
\text { noun phrase }\end{array}$ & Pre-Q & $\begin{array}{l}\text { morpheme preceding } \\
\text { the } Q \text { in a } \mathrm{NP}_{\mathrm{q}}\end{array}$ \\
\hline NPspec & specifying noun & Pron & pronoun \\
\hline NPspec/q & $\begin{array}{l}\text { phrase } \\
\text { quantified specifying } \\
\text { noun phrase }\end{array}$ & $\mathbf{Q}$ & $\begin{array}{l}\text { numeral plus classi- } \\
\text { fier; quantity ex- } \\
\text { pression (equivalent } \\
\text { to "Num + Clf") }\end{array}$ \\
\hline NPt ime & temporal noun phrase & Qt i me & $\begin{array}{l}\text { temporal quantity } \\
\text { expression }\end{array}$ \\
\hline NPyoc & vocative noun phrase & Quasi-Elab & quasi-elaborate ex- \\
\hline Num & numeral plus classi- & & $\begin{array}{l}\text { pression ( } 4 \text { syllables } \\
\text { but none repeated) }\end{array}$ \\
\hline & $\begin{array}{l}\text { fier; quantity ex- } \\
\text { pression (equivalent } \\
\text { to "Q") }\end{array}$ & QQ & $\begin{array}{l}\text { polyquantificational } \\
\text { expression (the } \mathrm{NP}_{\mathrm{Q}} \\
\text { contains more than } \\
\text { one Q) }\end{array}$ \\
\hline 0 & objec & $\mathrm{RC}$ & relative clause \\
\hline OV & $\begin{array}{l}\text { object plus verb; } \\
\text { object-verb } \\
\text { construction }\end{array}$ & RRC & $\begin{array}{l}\text { right-relative clause } \\
\text { (comes after head- } \\
\text { noun) }\end{array}$ \\
\hline
\end{tabular}


S

SE

SV

Vact

Vadj

Vadj-intens

$V_{h}$

$V_{h}+V_{h}$

$V_{h}+V_{v}$ sentence; subject

subordinate

expression

subject plus verb;

subject-verb

construction

verb

action verb

adjectival verb;

adjective

intensified adjective

verb head; main verb

two main verbs in

direct juxtaposition

("fortuitously

concatenated" verbs)

main verb modified by a post-head versatile verb (post-head concatenation)

$\begin{array}{ll}V_{\text {int }} & \begin{array}{l}\text { intransitive verb } \\ \text { (same as v.j.) }\end{array} \\ V_{t r} & \begin{array}{l}\text { transitive verb (same } \\ \text { as } v . t .)\end{array} \\ v V & \begin{array}{l}\text { pre-head versatile } \\ \text { verb }\end{array} \\ v V+V_{h} & \begin{array}{l}\text { main verb modified by } \\ \text { a pre-head versatile } \\ \text { verb (pre-head } \\ \text { concatenation) }\end{array} \\ V_{v} & \begin{array}{l}\text { post-head versatile } \\ \text { verb }\end{array} \\ V P & \text { verb phrase } \\ V P_{f} & \text { final verb phrase } \\ V P_{i n t e r j} & \begin{array}{l}\text { interjectory verb } \\ \text { phrase }\end{array} \\ V P_{n f} & \text { non-final verb phrase }\end{array}$

intransitive verb

transitive verb (same

pre-head versatile

main verb modified by a pre-head versatile verb (pre-head

concatenation)

post-head versatile

verb phrase

final verb phrase

interjectory verb

non-final verb phrase 
*A A is hypothetical or reconstructed; $A$ is ungrammatical

A > B A becomes B; A develops into B through time; B is the reflex of A

A $<$ B A is derived from B; A descends historically from B; B is the provenience of $\mathbf{A}$

$A \times B \quad A$ is an allofam of B; A and B belong to the same word-family

\begin{tabular}{|c|c|c|c|}
\hline ANT. & antonym & n. & note \\
\hline app. & apparently & nec'y & necessarily \\
\hline c. & century & neolog. & neologism \\
\hline cf. & compare & onomat. & onomatopoeia \\
\hline diff. & different; difference & pejor. & pejorative \\
\hline e.g. & for example & pl. & plural \\
\hline esp. & especially & poss. & possibly \\
\hline etym. & etymology; etymologically & prob. & probably \\
\hline etym. & obsc. etymology obscure & psm & passim \\
\hline euph. & euphemism & q.v. & which see \\
\hline ex. & example & $q q \cdot v$. & which see (pl.) \\
\hline fem. & feminine & sg. & singular \\
\hline fig. & figurative & $\operatorname{smn}$ & someone \\
\hline id. & same as the above & sthg & something \\
\hline i.e. & that is & stimes & sometimes \\
\hline infl. & influenced & syll. & syllable \\
\hline joc. & jocular & SYN. & synonym \\
\hline masc. & masculine & ult. & ultimately \\
\hline
\end{tabular}


